\title{
Test Migration for Efficient Large-Scale Assessment of Mobile App Coding Assignments
}

\author{
Farnaz Behrang \\ Georgia Tech \\ Atlanta, GA, USA \\ behrang@gatech.edu
}

\author{
Alessandro Orso \\ Georgia Tech \\ Atlanta, GA, USA \\ orso@cc.gatech.edu
}

\begin{abstract}
In recent years, there has been a growing interest in making education widely accessible using Internet technologies. Whether it is Massive Open Online Courses (MOOCs) or simply college courses offered to a large student population using an online platform, both education-focused companies and universities, often in collaboration with one another, have been investing massively in online education. The fact that hundreds, and more often thousands, of students take these online courses raises scalability challenges in assessing student assignments. In this paper, in particular, we present a technique (GUITESTMIGRATOR) that addresses the challenge of assessing mobile app coding assignments. Given a set of apps that implement the same specification, but can have completely different user interfaces, instructors normally have to manually run and check each app to make sure it behaves correctly and according to the specification. GUITESTMIGRATOR, conversely, allows for developing tests for one of these apps and automatically migrating these tests to the other apps, thus dramatically reducing the burden on the instructor. We implemented GUITESTMIgRATOR for Android apps and evaluated it on three sets of apps developed over three different semesters by students of an online graduate-level software engineering course. Our initial results show that our approach is promising and motivates further research in this direction. The paper also discusses possible applications of this approach for test evolution and test migration for real-world apps.
\end{abstract}

\section{CCS CONCEPTS}

- Software and its engineering $\rightarrow$ Software testing and debugging;

\section{KEYWORDS}

Automated assessment, test migration, mobile apps

\section{ACM Reference Format:}

Farnaz Behrang and Alessandro Orso. 2018. Test Migration for Efficient Large-Scale Assessment of Mobile App Coding Assignments. In Proceedings of 27th ACM SIGSOFT International Symposium on Software Testing and Analysis (ISSTA'18). ACM, New York, NY, USA, 12 pages. https://doi.org/10. $1145 / 3213846.3213854$

Permission to make digital or hard copies of all or part of this work for personal or classroom use is granted without fee provided that copies are not made or distributed for profit or commercial advantage and that copies bear this notice and the full citation on the first page. Copyrights for components of this work owned by others than the author(s) must be honored. Abstracting with credit is permitted. To copy otherwise, or republish, to post on servers or to redistribute to lists, requires prior specific permission and/or a fee. Request permissions from permissions@acm.org.

ISSTA'18, July 16-21, 2018, Amsterdam, Netherlands

() 2018 Copyright held by the owner/author(s). Publication rights licensed to ACM ACM ISBN 978-1-4503-5699-2/18/07 ..\$15.00

https://doi.org/10.1145/3213846.3213854

\section{INTRODUCTION}

In recent years, we have witnessed an increasing interest in making education more widely accessible by offering online courses in various formats and as part of different programs. As clear evidence of this, more than 700 universities [2] have partnered with about 30 education-focused companies [47], such as Coursera, edX, and Udacity, to offer courses either as Massive Open Online Courses (MOOCs) or as more managed online courses that are offered as part of both standard and ad-hoc degrees. Even without going to the extremes of MOOCs, whose average course is found to enroll around 43,000 students [29], online classes can have hundreds, when not thousands of students.

The high number of students taking online courses introduces many scalability challenges. One of the main challenges, in this context, is assessing student assignments [13, 34]. Because providing manual feedback is typically prohibitive for these large classes, different automated techniques have been proposed for grading student assignments (e.g., [21, 30-32, 50, 53]). Test-case based assessment, in particular, is the most common approach to assess student programming assignments [50].

This kind of approaches work well for programs with a well defined API (e.g., for low-level programming assignments) or with a very constrained GUI (e.g., a GUI where the set of widgets and their labels or IDs are fixed). Unfortunately, most non-trivial assignments that involve the creation of web or mobile apps consist in developing such apps based on some form of specification, and the students are free to define the GUI of the apps. In fact, developing the GUI of the app is sometime an integral part of the assignment. Grading this type of assignments necessarily requires manual effort, as each app must be checked manually and individually to make sure that it suitably implements the provided specification. Reducing such effort is thus particularly important, as market demand for developers of mobile apps is constantly growing, and online courses have become one of the most popular ways to learn mobile app development [6].

Our solution to this problem starts from the observation that apps developed by different students as part of a class project or an assignment might have different GUIs but are supposed to provide the same functionality (being based on the same specification). Therefore, the GUIs for these apps must share some commonalities, and such commonalities may allow for mapping elements across different GUIs, at least partially. Based on these observations, we propose a technique, called GUITESTMigRATOR, that operates as follows: given a set of GUI tests written for a reference app (source app), GUITestMigrator (1) maps the GUI of the source app to the GUI of other apps developed using the same specification (target apps) and (2) uses this mapping to transform the tests for the source 
app into tests for the target apps. More precisely, GUITEstMigRATOR takes as input a source app, a test case for the source app, and a target app. It then (1) analyzes and models the GUI events in the test case as a set of one or more test scenarios, (2) tries to match the scenarios to possible GUI event sequences in the target app, and (3) transforms the matched scenarios into actual test cases that can be run on the target app. When assessing student-developed apps, GUITESTMigRATOR allows instructors to develop tests for one of these apps and automatically migrate these tests to the other apps, thus dramatically reducing the burden of grading.

It is worth noting that, although several researchers defined techniques for repairing GUI test cases when an interface changes (e.g., [23, 41, 42]), most of these techniques assume and leverage the fact that the majority of the GUI is unchanged between the two app versions considered, which is not the case when migrating tests between different apps. The two problems thus require different approaches, and whereas test migration techniques could likely be used for test repair, the opposite is not true.

To evaluate our technique, we implemented it for Android mobile apps and test cases written using Google's Espresso testing framework [3]. We then used this prototype on three sets of apps developed by students of an online graduate-level software engineering course over three semesters. The apps within each set were developed independently by different teams of students provided with the same specification, which was different for every semester. The specifications were kept purposely vague, to avoid over-constraining the design and implementation of the apps. In fact, the GUIs of the student apps (see [5]) showed a large degree of variability. As test cases for our evaluation, we used the test cases that (some of) the teams developed for their apps.

Specifically, we evaluated GUITESTMigRATOR on 6 source apps per set (i.e., semester) and on 23, 20, and 20 test cases for the three sets, respectively. As target apps, we randomly selected a subset of the possible target apps: 65/97, 30/51, and 30/90 for the three sets. (We did not consider all the apps in the sets due to the cost of manually checking the results.) Given these inputs, GUITestMigrator migrated the test cases for the source apps into 43, 45, and 45 test scenarios and migrated them to the target apps. Overall, GUITESTMigrator was able to successfully migrate, on average, $78 \%$ of the GUI events in the scenarios, which corresponds to $68 \%$ of the test scenarios being fully migrated and $21 \%$ of the test scenarios being partially migrated. Among the unmatched events (22\%), 10\% did not have a counterpart in the target apps (true negatives), $8 \%$ had a possible counterpart, but GUITESTMIGRATOR was unable to find the match (false negatives), and $4 \%$ were incorrectly matched by GUITestMigrator (false positives). We believe that these results are promising and motivate further research in this direction, as discussed in Section 6.

This paper makes the following contributions:

- The novel idea of using test migration to support efficient large-scale assessment of mobile app coding assignments.

- A new technique that implements this idea for Android apps.

- An implementation of the technique for the Android platform that is publicly available, together with our experimental data and infrastructure [5].

- An initial empirical evaluation that shows the effectiveness and the potential usefulness of our technique.

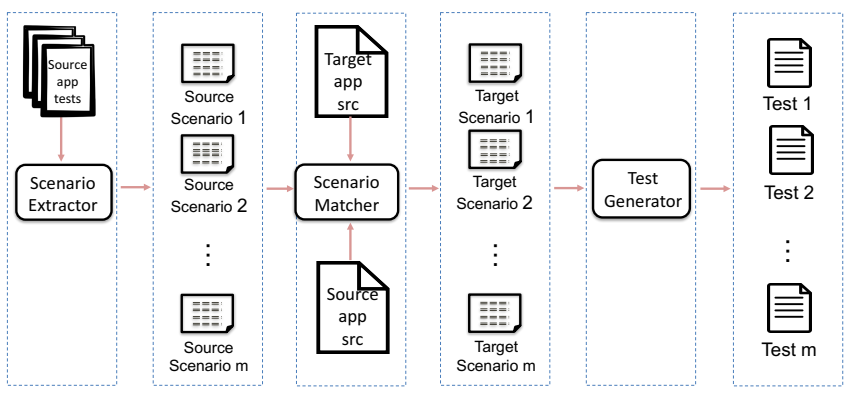

Figure 1: Overview of GUITESTMIGRATOR.

\section{MOTIVATING EXAMPLE}

As an example, assume we have a human-written GUI test case for a grocery list manager mobile app; the test verifies the app's functionality for "adding a new list", "renaming the list", and "deleting the list". Figure 2 shows the sequence of GUI events that the test case triggers to cover these scenarios. To add a list, the test clicks on the add button $(a \rightarrow b)$, types the name of the list $(b \rightarrow c)$, and clicks on the create button $(\mathrm{c} \rightarrow \mathrm{d})$. To rename the list, it first long-clicks on the name of the list, so that the rename option appears $(\mathrm{d} \longrightarrow \mathrm{e})$, and then clicks on the rename button $(\mathrm{e} \rightarrow \mathrm{f})$. At this point, there is an assertion in the test that checks whether the value of the EditText box is the same as the list name. If the assertion passes, the test continues by replacing the text with a new name $(f \rightarrow g)$ and then clicking on the rename button $(\mathrm{g} \rightarrow \mathrm{h})$. Next, there is another assertion that checks whether the name of the list has changed to the new name. If this assertion passes as well, the test deletes the list by long clicking on the new name, so that the delete option appears $(h \mapsto i)$, and then clicking on the delete button $(i j)$.

Given this sequence of GUI events for the above test and app (source app), GUITESTMigrator tries to automatically migrate these events to GUI events that can be consumed by another grocery list manager app (target app). Figure 3 shows the sequence of GUI events generated by GUITESTMIGRATOR for the target app. Although both apps belong to the same category, their GUIs are fairly different, which makes it non-trivial to perform the migration of GUI events.

In order to add a new list in the source app, one needs to click on the image button with a plus sign, while in the target app one needs to click on the button labeled "CREATE NEW LIST". To rename the list in the source app, one first needs to long-click on the list so that the rename option appears and can be clicked, whereas in the target app one has to skip the long-click and directly click on the edit image button (i.e., the button with the pencil symbol). Similarly, for deleting a list in the source app, one first needs to long-click on the list and then click on the delete button; in the target app, conversely, one can directly click on the trash image button. For this example, GUITestMigrator would be able to perform this migration and return to the user a test case with the sequence of GUI events that can be run on the target app.

\section{APPROACH}

Figure 1 shows an overview of our approach. At a high level, GUITestMigrator takes as input (1) a set of GUI test cases for the source app, (2) the source app, and (3) the target app, and produces as output a set of GUI tests for the target app. First, the Scenario 


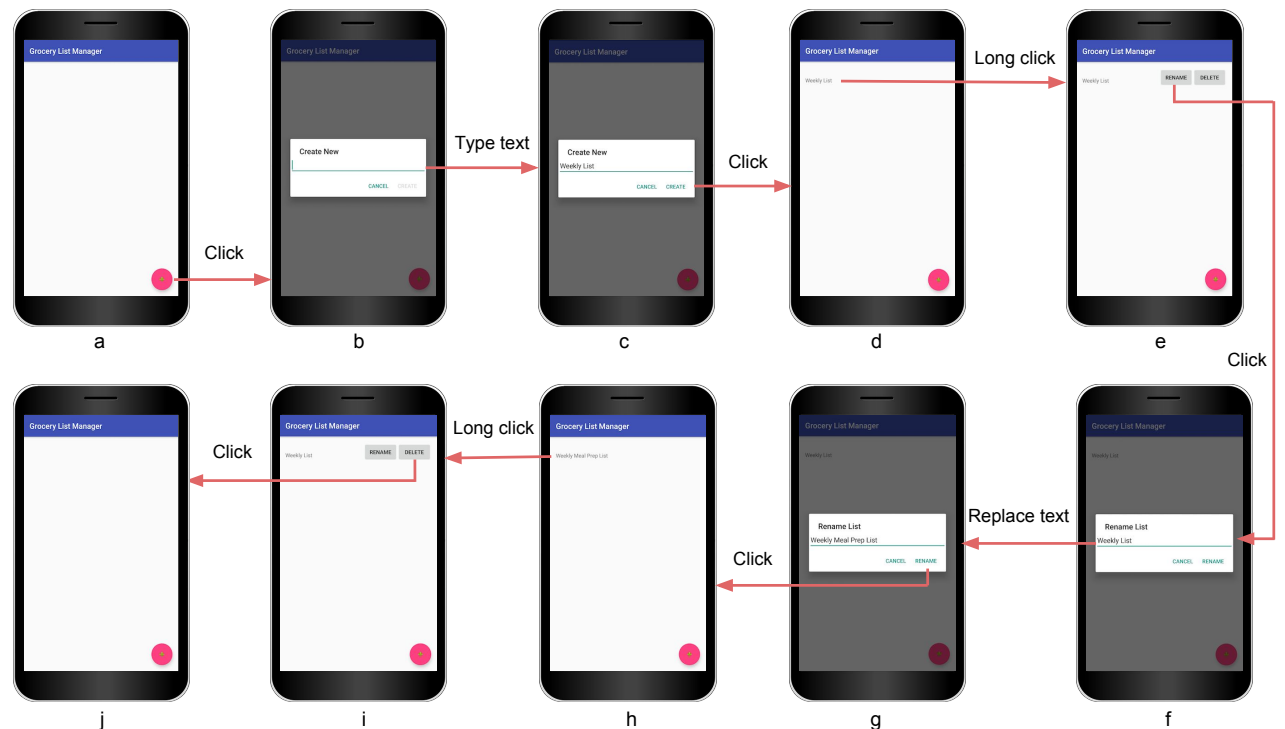

Figure 2: Sequence of GUI events triggered by the test case for the source app in our motivating example.

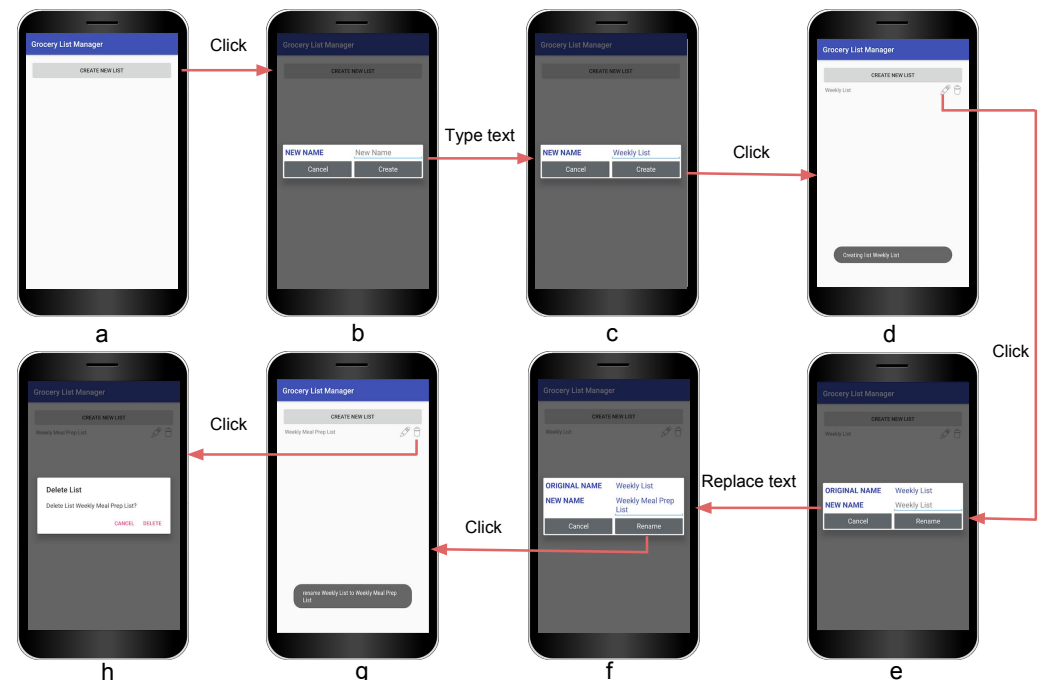

Figure 3: Sequence of GUI events migrated by GUITEsTMIGRATOR from the source app to the target app in our example.

Extractor extracts a set of test scenarios from the input test cases, where each scenario is a sequence of GUI events; each GUI event is a triple $a, t, i$, where $a$ is the action for the event (e.g., click), $t$ is the target of the action (e.g., a button), and (optionally) $i$ is an input value (e.g., the text for an EditText box). Then, given the source code of source and target apps, the Scenario Matcher is responsible for finding scenarios in the target app that match the test scenarios extracted in the previous step. Once the target scenarios have been identified, the Test Generator generates test cases for the target app, where each test case corresponds to a target scenario. We discuss each of these steps in detail.

\subsection{Scenario Extractor}

To migrate the test cases for the source app to the target app, GUITestMigrator divides each test case into a set of test scenarios. Specifically, it splits the sequence of GUI events in each test case into a set of smaller sequences of GUI events based on the assertions in the test case. Sequences of GUI events are often followed by assertions to test whether the outcome of triggering the GUI events up to that point is expected. Therefore, assertions can be used to understand the intent of the person who wrote the test and identify scenarios that test specific pieces of functionality.

Algorithm 1 describes how GUITESTMIgRATOR performs this step. First, it instruments the APIs in the testing framework, which provides information about GUI interactions. More precisely, the instrumentation records the actions performed by the test, the elements that are accessed, the input values that are used within the actions, and the assertions that are checked (line \#4). GUITESTMigrator then runs the tests and records the sequence of the GUI events that are triggered by each test method (line \#5). For each recorded trace that corresponds to a test method, GUITESTMigrator locates the start and end of the method, as well as GUI 


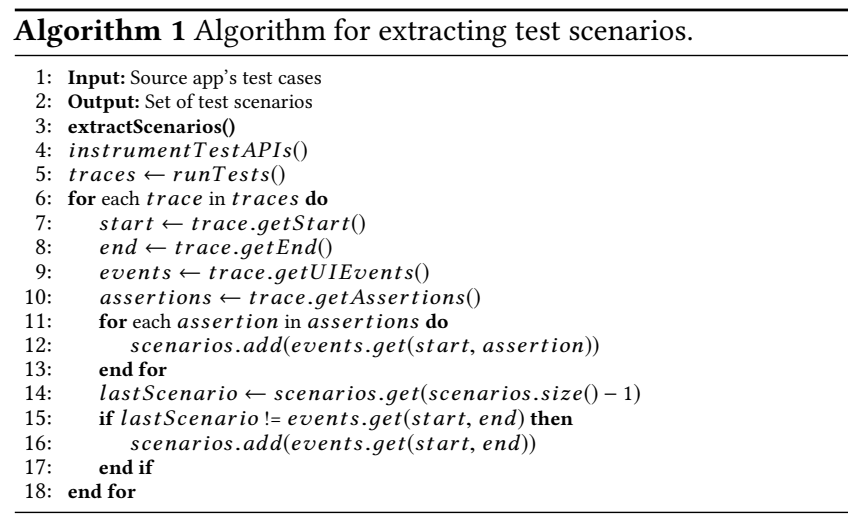

events and assertions (lines \#7-10). GUITESTMIGRATOR considers the sequence of events from the beginning of the test method to each assertion as a separate scenario (lines \#11-13). It then checks whether there are any extra events that are not followed by an assertion and would therefore be discarded; if so, GUITESTMIGRATOR also generates an additional scenario that consists of the whole method, from beginning to end (lines \#14-17).

Considering our motivating example, there are two assertions in the test case. The first one occurs when the test reaches screen $f$ and checks whether the value of the EditText box and the list name are the same. The second assertion occurs after rename is triggered, on screen $h$, and checks whether the name of the list has changed. Therefore, GUITESTMIgRATOR would split the test case into three test scenarios: $\mathrm{a} \longrightarrow \mathrm{f}, \mathrm{a} \longrightarrow \mathrm{h}$, and $\mathrm{a} \longrightarrow \mathrm{j}$ (which can be seen in figure 2).

\subsection{Scenario Matcher}

Given (1) the source code of source and target apps and (2) the test scenarios extracted by the scenario extractor, the Scenario Matcher tries to find sequences of GUI events in the target app that match each scenario, as described in Algorithm 2.

For each test scenario, GUITESTMigRATOR first gets the sequence of GUI events in the scenario (source GUI events, hereafter) and launches the target app (lines \#7-8). It then starts crawling the app to find a match in the target app (target GUI event) for each source GUI event. This step terminates when either GUITEstMigRATOR finds matches for all the source GUI events or the exploration reaches a predefined time threshold (line \#9). In the rest of the discussion, we use the term screen to indicate a collection of GUI elements in a given state, and the term interactable element to indicate a GUI element that can be the target of a user action. To find a match, GUITESTMIGRATOR gets the GUI hierarchy of the current screen and finds all the interactable elements in the screen. To do so, it traverses the GUI hierarchy and checks the attributes of the nodes therein. Based on the value of the attributes for the nodes or their ancestors, the algorithm flags as interactable the nodes that are clickable, long-clickable, or checkable (lines \#10-11).

Because, while crawling the app, the algorithm can visit identical or almost identical screens multiple times, GUITESTMIGRATOR encodes each screen visited as a set of features, where the features consist of all the nodes in the screen's GUI hierarchy together with their attributes. For each new screen encountered, the algorithm then computes the cosine similarity of this screen with that of previously visited screens in terms of these features. If this similarity is above a given threshold, the algorithm considers the screens to be equivalent and reuses the previously computed information about interactable elements.

Once it has identified all the interactable elements in the current screen, by either newly computing or reusing them, GUITESTMIGRATOR compares each element against the source GUI events that have not been already matched and tries to match it with an event that appears as early as possible in the sequence (lines \#12-31). In other words, GUITESTMIgRATOR tries to find matches for the sequence of source GUI events in the order in which they appear, while allowing for some events not to be matched. Given a target GUI event $t$, a source GUI event that matches $t$ with a lower similarity score (above the defined threshold) but appears earlier in the sequence is therefore considered a better match for $t$ than a source GUI event with a higher similarity score that appears later in the sequence (we discuss how we calculate the similarity score in Section 3.3). To illustrate this on the motivating example, consider the first three source GUI events: \{Click, Add Button, Null\}, \{Type text, EditText, "Weekly List"\}, and \{Click, Create Button, Null\}. Let us assume that GUITESTMIGRATOR successfully matches the first screen of the target app. It would then compare the interactable elements of the second screen (i.e., Create Button, Cancel Button, and New Name EditText) against the second and third source GUI events mentioned above. Among all possible matches, Create Button matches the third source GUI event \{Click, Create Button, Null\} with the highest similarity score. Nevertheless, GUITestMigrator considers \{Type text, EditText, "Weekly List"\} as a better match because it appears earlier in the sequence, despite the fact that the similarity score of the match is lower.

To perform the matching we just described, GUITESTMigRATOR processes all the unmatched events whose index is lower than mIndex, the index of the last matched event in the sequence of source GUI events (lines \#12-17). It then calculates a similarity score between 0 and 1 for each possible match and, because test scenarios might share GUI events, caches the score for later reuse (lines \#1823). If the similarity score is higher than both the matching threshold and the highest score obtained so far, GUITESTMIGRATOR considers this match to be valid until a better match is possibly found (lines \#24-28). In this process, the algorithm may match a GUI event with index $i_{j}$ which is lower than the indexes $i_{l}, \ldots, i_{n}$ of source GUI events already matched. In this case, GUITESTMIGRATOR invalidates all the matches from the one for event $i_{j+1}$ to the one for event $i_{n}$ and continues the search from the last matched event (lines \#41-47). In addition, the algorithm marks all the target GUI events whose matches have been invalidated as random events (line \#48). In this way, these events will be treated, during test generation, like the events actually added through random search, as described in the next paragraph. Note that these events cannot be simply eliminated right away because they may still be needed to reach the screen in which $i_{j}$ can be matched.

It could also be the case that the algorithm does not find any match for the current screen. In this case, GUITESTMIGRATOR backtracks to a previous screen with multiples possible matches having similarity score above the threshold, if any, and explores an alternative match (with a lower score than the one previously selected) there. If no such screen exists, the algorithm semi-randomly picks one of the not-yet-selected interactable elements (lines \#32-38). By semi-randomly, we mean that GUITESTMigrator takes into 
account the structural relationships between the elements when deciding which interactable elements to select. Assume that there are two forms $f_{a}$ and $f_{b}$ in a screen, each containing an EditText box and a submit button, and that the previous match resulted in typing an input into an EditText box in form $f_{b}$. In this case, GUITestMigrator's semi-randomly selection would pick the submit button in $f_{b}$ as the next element because it is semantically related to the previously selected element. To identify structural relationships between elements, GUITEstMigrator leverages their XPath [9] in the GUI hierarchy of the screen, by favoring elements whose XPath shares longer subpaths with the XPath of the previously selected element. When picking elements this way, GUITESTMIGRATOR also marks them as random (line \#38), while it marks them as non-random otherwise (line \#40). As we discussed above, this distinction is important during test generation, to eliminate unnecessary extra events (see Section 3.4).

Once the next event to trigger is found, whether as an actual match or by semi-random selection, GUITESTMIGRATOR adds the event to the list of target GUI events, marks the corresponding source GUI event as matched, and triggers the event (lines \#51-54).

Finally, when the algorithm terminates, it adds the sequence of target GUI events as a scenario to the list of target scenarios (line \#56). This scenario can either be a complete one, if GUITESTMIGRATOR was able to match all source events, or a partial one, if the algorithm terminated due to a timeout.

\subsection{Computing Similarity Score}

As we discussed in Section 3.2, GUITESTMigrator matches source and target GUI events using a similarity score that ranges between 0 to 1 . GUITESTMIGRATOR computes this similarity score based on two criteria: the action associated with the event and the target of such action. First, GUITestMigrator checks whether the action associated with the source GUI event exists for the target GUI event and, if it does not, assigns zero to the similarity score for the two events. Considering the motivating example, for instance, the action associated with the fourth source GUI event is long-click, and this action does not exist for any of the interactable elements of the target app. GUITESTMIGRATOR would therefore assign zero to the similarity between this event and any of the interactable elements. Conversely, if the action associated with the source GUI event exists for the target GUI event, GUITESTMIgRATOR compares the target of the action for the two events. If the target element is of a type that accepts textual input, GUITESTMIgraTOR checks whether both target elements are of the same type and assigns a zero similarity score otherwise. In other cases, the types can be different, as different widgets, such as a Button, an ImageButton, and a TextView in Android, can provide the same functionality.

Next, GUITESTMigrator considers the text associated with the target elements, if any. (If an element has no text associated with it, GUITESTMIgRATOR simply skips it.) Some elements have a label, which makes it straightforward to extract the text associated with them. This is the case for the button on screen $a$ of the target app in the motivating example, for which GUITESTMIgRATOR would extract the text "create new list". In other cases, a target element may still have text associated with it, but in a more subtle way. To account for these cases, GUITEstMigrator distinguishes between image and non-image elements. Specifically, for non-image

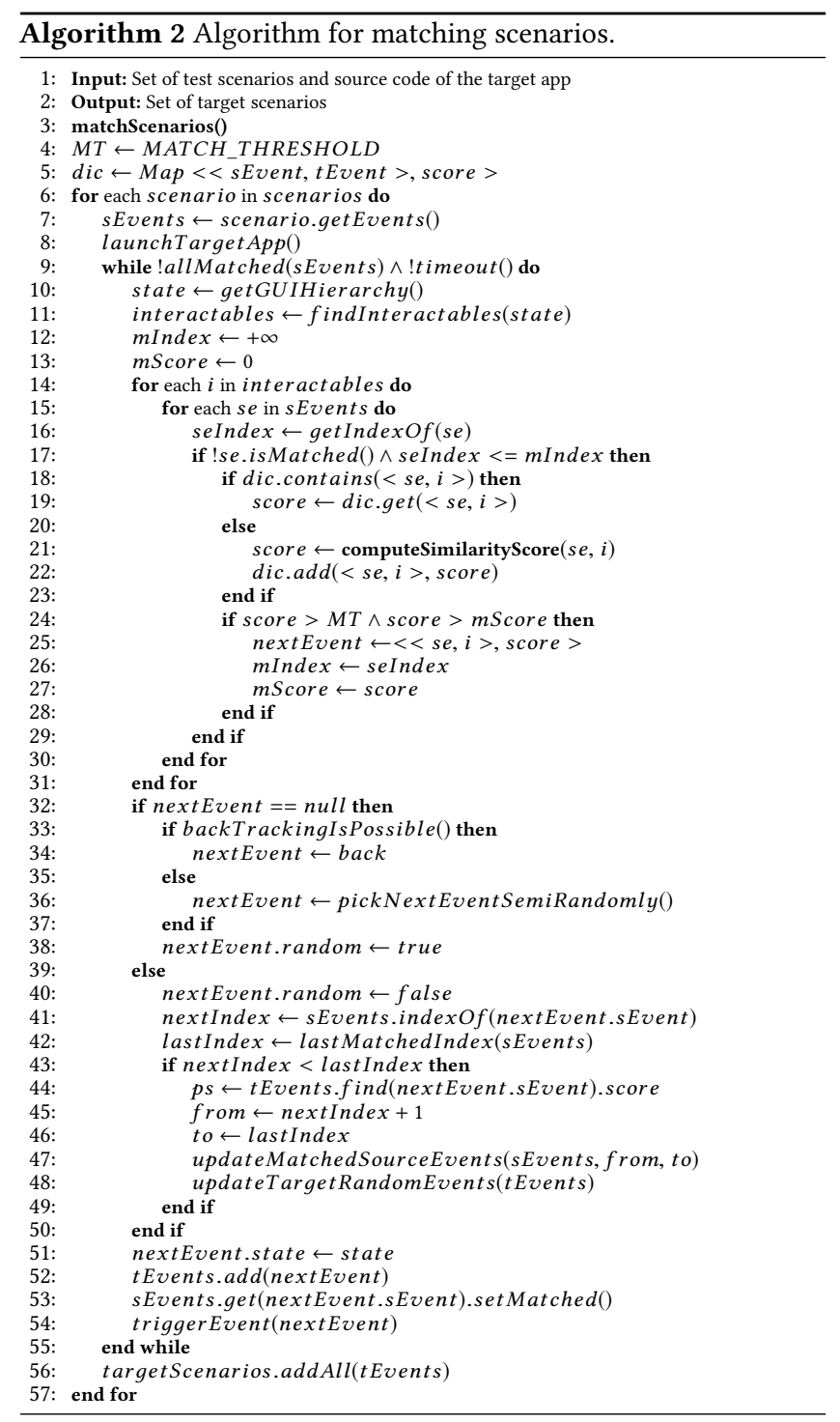

elements, GUITestMigrator looks for labels in the vicinity of the element by considering its XPath in the GUI hierarchy, where vicinity is defined in terms of traditional distance of the elements and based on a given threshold. In the motivating example, for instance, the target element for the second source GUI event on screen $b$, \{Type text, EditText, "Weekly List"\}, does not have any directly associated text; GUITESTMIGRATOR would still identify "Create New" as the text associated to the element because that label is right above such element. For image elements, conversely, GUITESTMIGRATOR converts the image associated to the element to text by leveraging metadata-based image retrieval techniques (e.g., [48]). As these techniques typically do, GUITESTMIGRATOR treats the problem of image retrieval as one of text retrieval, in which images are described by some metadata (e.g., alternate text, descriptions, or image filenames). More precisely, GUITESTMIGRATOR first checks whether the developer provided a content description for the image, by parsing its GUI hierarchy and extracting the value of the corresponding attribute, if present. To illustrate with an example, 
this is how GUITESTMigrator would extract the text associated with the image on screen $d$ of the target app in Figure 3, which is matched with source GUI event \{Click, Rename Button, Null\}. If the content description attribute of the element does not have any value, GUITESTMigrator takes advantage of the image filename. The rationale for this choice is that image filenames in mobile apps tend to be meaningful and to encode various information, such as functionality and visual appearance of the corresponding element. Based on our analysis of thousands of default images in Android apps, we defined a parser that preprocesses image filenames to extract from them relevant information. In our motivating example, for instance, the filenames of the images in the source app's screen $a$ and in the target app's screen $d$ are ic_input_add and ic_menu_delete, respectively; GUITESTMIgRATOR would extract text "add" from the former and text "delete" from the latter.

After extracting the text associated with both target elements, GUITESTMigRATOR does some preprocessing before performing the text comparison. The preprocessing involves tokenizing the text, applying lemmatization (i.e., full morphological analysis to accurately identify the lemma for each word), and using a part-ofspeech (POS) tagger to assign a category to each word (e.g., noun, verb). The POS tags are then used to discard unimportant parts of the text, such as pronouns, conjunctions, and prepositions. After this preprocessing, GUITestMigrator uses one of the $W u$ and Palmer (wup) semantic similarity based measures to calculate the text similarity score [54]. We chose a semantic similarity measure because two terms can be semantically similar (e.g., can be synonyms or hypernyms) even if they are lexicographically different, and we specifically chose the wup measure because of its simplicity and efficiency [51]. Given two words, wup computes relatedness by considering (1) the depths of the two synsets (i.e., group of synonyms) in the WordNet [43] taxonomies for the two words and (2) the depth of the least common subsumer (LCS)-the common ancestor deepest in the taxonomy that connects the two words.

As WordNet is a widely used general purpose lexical database, and GUITEstMigrator is used in the domain of mobile apps, we enriched the database by adding some words related to the domain knowledge of mobile apps. To do so, we manually classified thousands of default images in Android apps based on their similarity. (These filenames for similar images are not necessarily identical or related in general, but they are related and could be used interchangeably in the domain of mobile apps.) To illustrate, consider two images with a plus sign, one named "add" and the other one named "create"; in the context of Android apps they belong to the same category and would therefore be in the database. This information actually helps GUITESTMIGRATOR in our motivating example, when matching the first source GUI event, \{Click, Add Button, Null $\}$ with button "Create New List" in the first screen of the target app. To further extend WordNet with domain-specific information, we also added to it words such as "OK" and "Confirm", that are commonly being used interchangeably in mobile apps.

Using our extended WordNet database, GUITestMigrator considers all the tokens in the text for the source and target elements and matches each source token with the most similar target token according to the wup measure. It then computes the overall similarity score by averaging the score of the matched tokens.

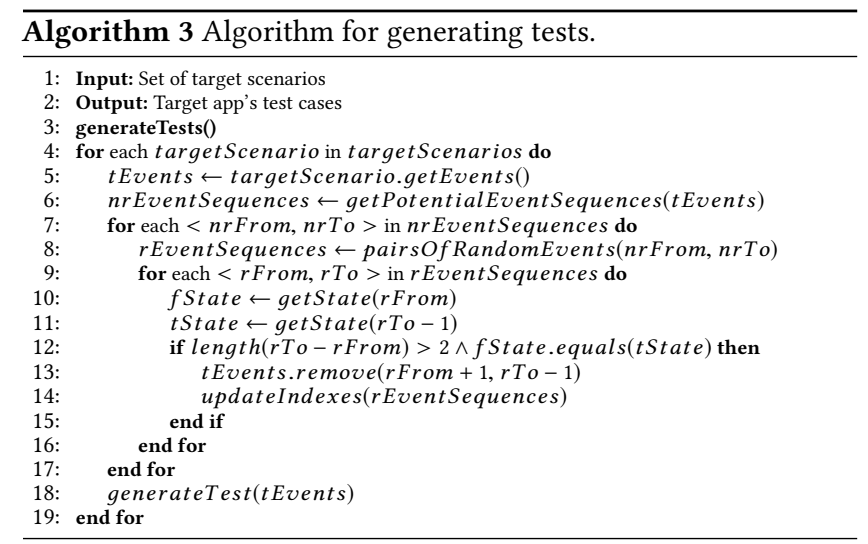

\subsection{Test Generator}

In this step, GUITESTMIgRATOR takes the set of target scenarios produced by the previous step and generates test cases for them. As discussed in Section 3.2, not all of the events selected during scenario matching must be part of the test cases, as it may be possible to safely eliminate some of the events marked as "random". The reason for this is that GUITESTMIGRATOR might have added these random events unnecessarily during crawling. As an example, consider: (1) a source app $S A$ with an "add" button that creates a new item of some sort; (2) a test case $T$ for $S A$ that simply creates two items by clicking on the "add" button twice; and (3) a target app TA that also has an "add" button that creates a new item but also switches to a second screen with a "back" button that returns to the initial screen and a "noop" button that does nothing. When migrating $T$ from $S A$ to $T A$, GUITESTMigrator would easily map the first click to "add" in $S A$ to a corresponding click to "add" in $T A$, which would take $T A$ to its second screen. At this point, the random exploration may result in one or more clicks to the "noop" button before correctly clicking the "back" button (and then mapping the second click to "add"). Any click to the "noop" button could clearly be discarded when generating the actual test for $T A$, as the only random event needed is the clicking of the "back" button.

Algorithm 3 presents the test minimization approach used by GUITESTMigrator. The algorithm first identifies potential subsequences of target GUI events for which minimization might be possible; minimization is only possible for those subsequences in which there are two non-random GUI events with at least one random GUI event in between (lines \#5-6). The ideal case is one in which all random GUI events between non-random GUI events can be eliminated. In most cases, however, this is not possible. To identify which random events can be safely removed in a subsequence, the algorithm checks whether the app state after triggering the first event in the subsequence and the app state before triggering the last event in the subsequence are the same for all subsequences with a minimum length of three (starting from the longest ones) (lines \#7-12). If so, it removes all the GUI events in the subsequence and updates the overall sequence accordingly (lines \#13-14). (To check whether two states are the same, the algorithm compares their GUI hierarchies and checks whether they are identical.) Once the process for a target scenario is done, a test is generated for the updated sequence of target GUI events (line \#18). 


\section{EVALUATION}

To assess the usefulness and effectiveness of our technique, we implemented GUITESTMIgRATOR and performed an empirical evaluation in which we investigated the following research questions:

(1) RQ1: How accurate is GUITESTMIGRATOR in migrating GUI events (i.e., test inputs) from source to target apps?

(2) RQ2: How long are the sequences of GUI events successfully migrated by GUITESTMIGRATOR?

(3) RQ3: What limitations of GUITESTMIGRATOR prevent it from successfully migrating all GUI events?

\subsection{Implementation}

We implemented GUITestMigrator for Android apps and test cases written using the Espresso testing framework [3] because (1) Android is the most popular mobile app platform, and (2) Espresso is widely used and actively maintained by Google. Moreover, Espresso is fully integrated within Android Studio [1], which supports test development (e.g., using test recorders). We modified Espresso to enable collection of relevant dynamic information during test execution. Note that our implementation requires the source code of source and target apps because that is a requirement of Espresso; switching to a different framework (e.g., UIAutomator [7]) would remove this requirement. More generally, our approach is not specific to the Android platform and Espresso and could be ported to other mobile platforms and testing frameworks.

\subsection{Evaluation Setup}

To evaluate GUITestMigrator, we used three sets of mobile apps developed by teams of students of an online graduate-level software engineering course during three different semesters: GoBowl, an app for managing a bowling alley; GroceryListManager, an app for creating and editing grocery lists; and TCCart, an app for handling payments and rewards for coffee carts. Each set of apps was developed based on the high-level requirements provided by the instructor. As an example, we provide one of the requirements for the GroceryListManager app: "A grocery list consists of items the users want to buy at a grocery store. The app must allow users to add items to a list, delete items from a list, and change the quantity of items in the list (e.g., change from one to two pounds of apples)." The complete requirements for all three apps are available online [5] Most of the students enrolled in the course had years, when not decades, of professional software development experience, which should result in a more realistic development scenario.

We examined all the apps developed by the students to identify those that had Espresso tests. (Students were not required to write tests in Espresso format, so only a subset of apps had them.) We then randomly selected 6 source apps per set (18 overall) among those that we were able to run successfully. Overall, we were able to collect 23 tests for GoBowl, 20 tests for GroceryListManager, and 20 tests for TCCart. We then used GUITESTMigRATor to migrate each of these tests from the corresponding source app to a set of randomly selected target apps: 30 out of 51 total apps for GoBowl, 65 out of 97 for GroceryListManager, and 30 out of 90 for TCCart. Note that (1) we did not consider all the apps in the sets due to the cost of manually checking the results and (2) we used a different number of target apps for GroceryListManager because it was the only app considered in an initial version of the evaluation.

\subsection{RQ1: Accuracy}

To answer RQ1, we manually inspected the GUI events migrated by GUITESTMigRATOR from source to target apps and labeled each source GUI event migration in one of four ways: correct match, incorrect match, not matched (not exist), or not matched (exist). A correct match (resp. incorrect match) is basically a true positive (resp., false positive): an event migration in which triggering the source and target events have (resp., does not have) the same observable effect. A not matched (not exist) (resp., not matched (exist)) is basically a true negative (resp., false negative): a case in which GUITESTMIGRATOR is unable to map a GUI event in the source app to a corresponding event in the target app, and such an event does not exist (resp., does exist).

Figure 4 shows the percentage of migrations of each of these four types for the scenarios in the three apps considered; the $x$ axis represents the scenarios in ascending order of number of GUI events. As the figure shows, the sum of the percentages of true positives and true negatives (i.e., correct match and not matched (not exist)) is above $80 \%$ for all the scenarios with length under 19 , except for Scenario \#12 in the Grocery List Manager apps, for which it is $73 \%$. (The last part of that scenario involves a search for an item in a grocery list, which is performed in a completely different way with respect to all other apps.) Conversely, the percentages of false positives and false negatives (i.e., incorrect match and not matched (exist)) for the same scenarios (length under 19) range from $0 \%$ to $9 \%$ and from $0 \%$ to $21 \%$, respectively. For the scenarios of length 19 and above, the sum of the percentages for correct match and not matched (not exist) is still above 70\%, except for the last two scenarios in the GroceryListManager (lengths 34 and 35) and TCCart apps (length 44 and 45), where the percentages are 69\%, $67 \%, 66 \%$, and $68 \%$, respectively. The percentage of incorrect match migrations for the same scenarios (length 19 and above) ranges from $5 \%$ to $11 \%$, and that of not matched (exist) migrations ranges from $11 \%$ to $27 \%$.

In summary, GUITEstMigrator successfully migrated, on average, $78 \%$ of the GUI events in each scenario. Of the remaining $22 \%, 10 \%$ did not have a counterpart in the target apps (true negatives), $8 \%$ were missed by GUITestMigrator (false negatives), and $4 \%$ were incorrectly matched (false positives). We can therefore conclude that GUITESTMIgRATOR was accurate for $88 \%$ (78\% true positives $+10 \%$ true negatives) of the GUI events.

\subsection{RQ2: Sequences Length}

To answer RQ2, we studied the length of the GUI event sequences successfully migrated by GUITESTMigrator. Figure 5 shows this information. The dots in the figure represent the length of the longest sequence of source GUI events that were correctly migrated by GUITESTMigrator, and the size of the dots is proportional to the number of target apps whose longest sequence is of that length.

For the three apps considered, the range of values for the length (len) of the longest sequences, its median (med), and its standard deviation ( $s t d$ ) are as follows: for GoBowl, len between 1 (Scenarios $\# 1$ and \#2) and 17 (Scenario \#43), med between 3 and 11, and std between 0.3 and 3; for GroceryListManager, len $\mathrm{b} / \mathrm{w} 0$ (Scenarios \#13 and \#14) and 30 (Scenarios \#40-\#45), med b/w 3 and 13, and std $\mathrm{b} / \mathrm{w} 0$ and 6; and for TCCart, len $\mathrm{b} / \mathrm{w} 1$ (Scenario \#2) and 28 (Scenario \#42), med b/w 3 and 14, and std b/w 0.5 and 5. 


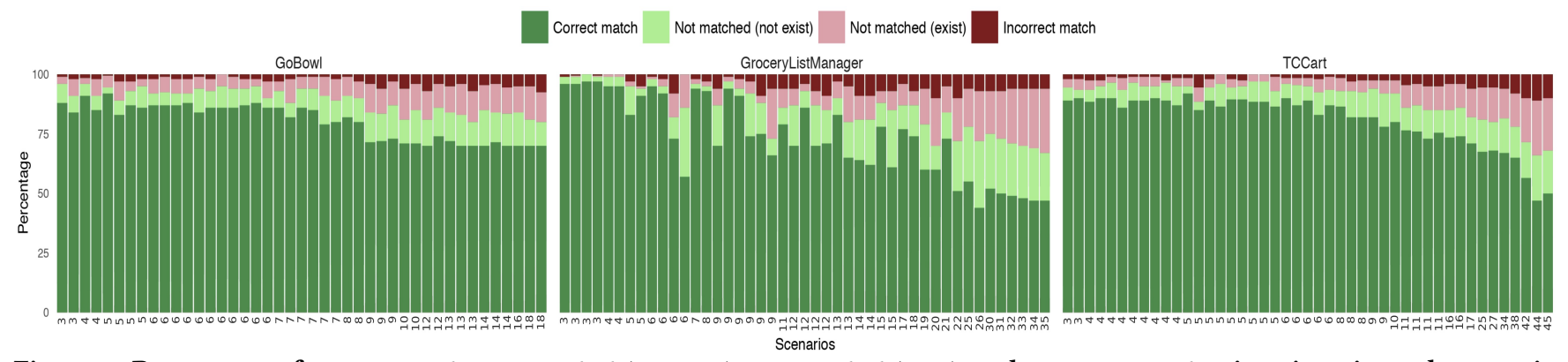

Figure 4: Percentage of correct match, not matched (not exist), not matched (exist), and incorrect match migrations in each scenario. The $x$ - axis represents the scenarios in ascending order of number of GUI events.
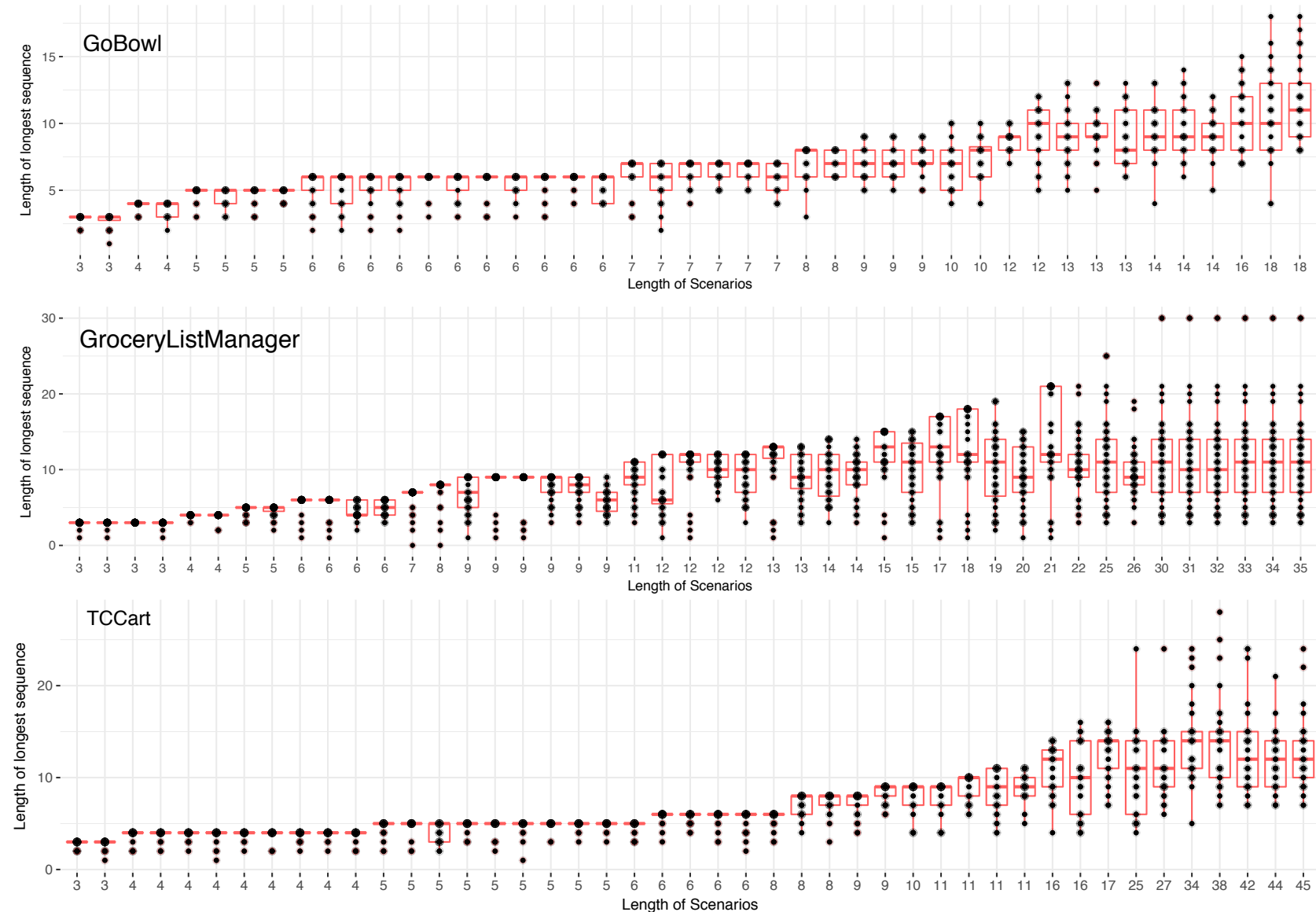

Figure 5: Longest sequence of correctly matched GUI events for each test scenario and target app.

Figure 6 provides a different view of this data by showing a heat map of the distribution of the correctly matched GUI events for each scenario. In the figure, the $x$-axis represents the GUI events in the source scenarios, and the $y$-axis represents the scenarios considered. We use a spectrum of colors to represent the percentage of target apps for which that event is correctly migrated in each scenario-the higher the percentage, the darker the color. (As shown in the legend, the figure uses 10 different hues, each corresponding to a $10 \%$ range of values.) The following table summarizes the information in the figure, by showing how many source GUI events GUITESTMIGRATOR successfully migrated for three sets of apps considered, in terms of percentages of target apps (e.g., for over $90 \%$ of the GoBowl apps, GUITESTMIgRATOR was able to migrate $63 \%$ of the GUI events).

\begin{tabular}{|l|r|r|r|r|}
\hline App set & $>90 \%$ apps & $70-90 \%$ apps & $40-70 \%$ apps & $<40 \%$ apps \\
\hline \hline GoBowl & $63 \%$ & $20 \%$ & $16 \%$ & $1 \%$ \\
\hline GroceryListManager & $50 \%$ & $25 \%$ & $20 \%$ & $5 \%$ \\
\hline TCCart & $56 \%$ & $24 \%$ & $13 \%$ & $7 \%$ \\
\hline
\end{tabular}

Overall, on average over all scenarios, GUITESTMIGRATOR successfully migrated the longest possible sequence (i.e., the complete sequence minus the source GUI events for which there was not a corresponding target event) for $60 \%$ of the target apps. 

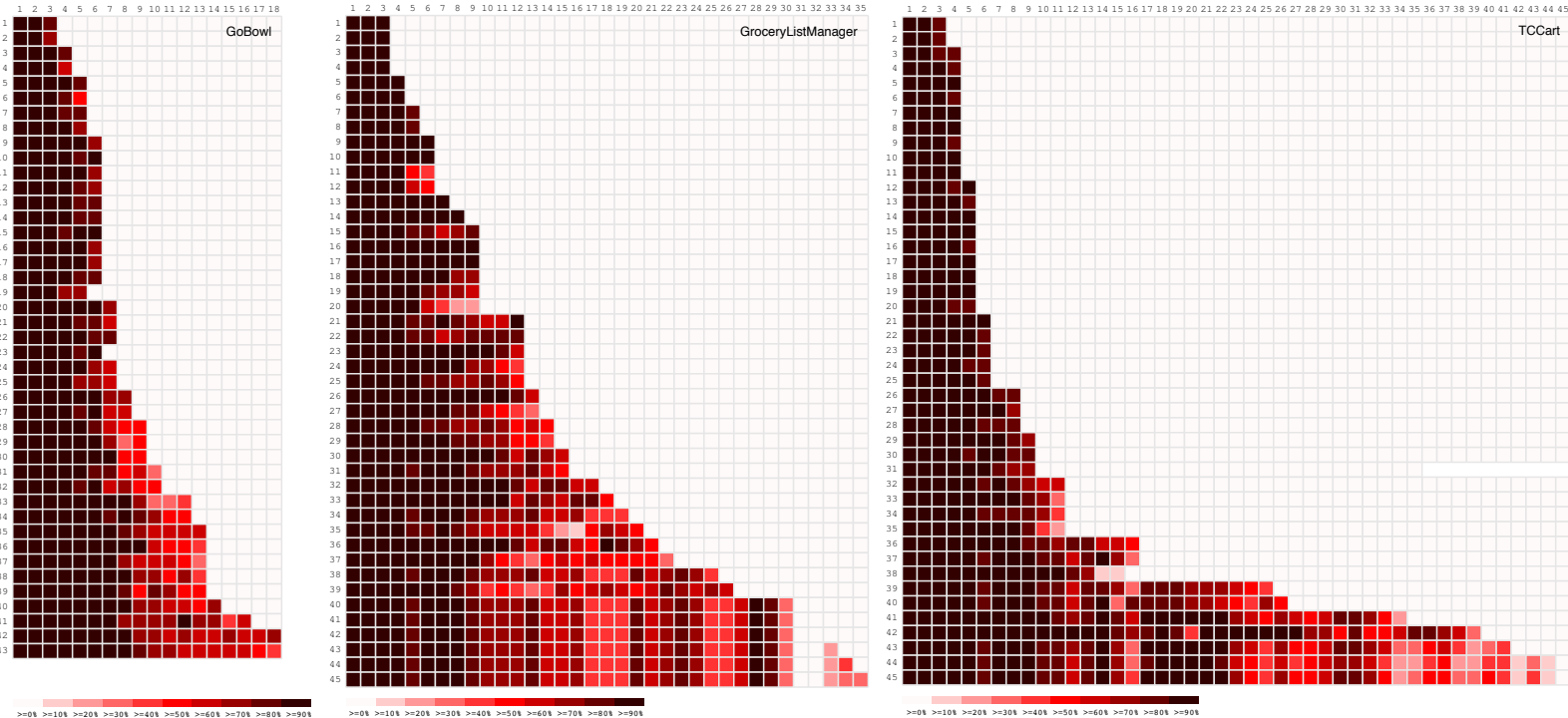

Figure 6: Distribution of correctly matched GUI events for each scenario. The $\boldsymbol{x}$-axis represents the source GUI events, whereas the $y$-axis represents the scenarios.

\subsection{RQ3: Categories of Unsuccessful Transformations}

To answer RQ3, we manually inspected the root causes of all unsuccessful migrations, that is, all the cases labeled not matched (exist) and incorrect match in all scenarios. We identified five categories of root causes (plus a catch-all category), which we present together with the percentage of unsuccessful transformations for each category.

Using different GUI events for performing a task (30\%). This is the largest category and consists of cases in which performing a task requires considerably different types of GUI events in the source and target apps. To mitigate this issue, we could extend GUITEsTMigrator's dynamic exploration so that it takes into account (1) possible future states of the app and (2) events enabled in these states. We could do this by statically modeling the target app as a state-flow graph (SFG), in which nodes represent states of the app and edges represent event-driven transitions between them [55].

Getting lost during dynamic exploration (28\%). GUITESTMigRATOR can get lost during its dynamic exploration of a target app, especially when source and target apps are considerably different and there is a large number of source GUI events. To address this problem, GUITESTMigrator could use an SFG to prune the search space by avoiding potentially useless states.

Matching missing GUI events (13\%). To illustrate this category, let us consider our motivating example and imagine a scenario in which a list is deleted. After selecting the delete button, the app might either ask the user to confirm the deletion or immediately delete the list. In this case, if the source app does the former and the target app does the latter, searching for a GUI event corresponding to a confirmation in the target app is not only unnecessary, but also problematic. To address this issue we could, also in this case, compute and use an SFG to perform a look ahead when matching events. If an event cannot be matched, but the subsequent one can, GUITEstMigRATOR could then simply drop the unmatched event.

Using widgets with unusual labels (12\%). This category involves widgets that utilize "unusual" labels. To illustrate, let us consider the sequence of GUI events for renaming a list in our motivating example. After typing the new name for the list, the next expected GUI event is a click on a confirmation button with a label such as "confirm", "yes", or "ok". However, one of the target apps uses a button with label "enter", which GUITESTMigrator is unable to match. In this case, there is a tension between making the lexical databases even richer, so that they can handle all these cases, and matching terms that are actually unrelated. One possibility would be to extend the lexical database on demand: a term that was not matched but should have been would be added to the database for future use, whereas a term that was not matched because it was poorly chosen would be ignored.

Using predefined lists (10\%). When using predefined lists of items in a menu (e.g., a list of groceries in the GroceryListManager app), a selected item from a list in the source app might not exist in the target app. In this case, that particular GUI event (i.e., selecting the item from the list) would be affected during migration, and subsequent GUI events that depend on it may be affected as well. Although the current version of GUITESTMIgRATOR mitigates this problem by generalizing the name of items using hypernyms based on the WordNet dictionary (e.g., "apple" to "fruit"), this approach is not enough in all cases. To further mitigate this issue, we will consider additional approaches, such as selecting random elements from a list when a specific one cannot be found.

Others (7\%). This category include those issues (7\%) that are very specific to the apps considered and cannot be easily generalized.

\subsection{Threats To Validity}

The main threat to the external validity of our results is that they were computed by considering a limited set of apps and may thus 
not generalize. Based on our experience and understanding of the problem space, however, we do not expect to find dramatic differences in the way GUITESTMIGRATOR would work for different sets of student apps. As for threats of internal validity, we manual inspected all of our results and are therefore fairly confident that they are correct.

\section{RELATED WORK}

The work most closely related to ours is in the areas of automated grading, GUI test repair, and GUI test generation.

\subsection{Automated Grading}

There has been a large body of research on automated grading, dating as far back as 1960 [26]. Ihantola and colleagues [28] present a survey of various systems developed for automated grading of programming assignments (e.g., [10, 15, 19, 49, 50]). The majority of these approaches focus on introductory computer programming courses and check the correctness of a student's solution by running it against a set of test cases. Some of the auto-grading concepts presented in the literature have also been integrated into commercial tools, such as Vocareum [8] and Gradescope [4]. None of these existing approaches, however, supports GUI-based apps and, most importantly, can perform test migration, as our work does.

\subsection{GUI Test Repair}

Memon [41] proposes a technique that automatically repairs GUI test suites for regression testing. GUITAR [42] repairs test cases that have become unusable due to changes in a GUIs. SITAR [23] is another technique that repairs GUI test scripts written for an earlier version of the same software by using annotated event-flow graphs, a set of transformations, and human input. Grechanik and colleagues propose a GUI test repair technique that automatically identifies changes between GUI objects and uses this information to help testers perform manual repair [24]. ATOM [35] automatically maintains GUI test scripts for evolving mobile applications. Daniel and colleagues propose an approach in which GUI changes are recorded and later used to repair test cases [18]. Huang and colleagues use genetic algorithms to both repair broken GUI test cases and generate new tests [27]. TIGOR is a tool that uses static analysis to help testers determine type errors in GUI scripts [22]. Zhang and colleagues present a technique that uses static and dynamic analysis, along with random testing, to automatically repair broken workflows for evolving GUI applications [57]. The technique by Li and colleagues helps GUI editors map source code to GUI views when code changes [33]. Many researchers propose various approaches to support migration of legacy GUI tests to modern GUI systems by reverse engineering user interfaces (e.g., [20, 36, 52]). WATER uses differential testing to suggest repairs for broken test scripts for web applications [17]. Finally, Omari and colleagues propose a technique for generating XPath extraction queries for a family of websites that contain the same type of information [44, 45]. As we also stated in the Introduction, our work differs from these approaches because it migrates test cases between apps, rather than repairing during software evolution. In fact, these techniques could not be readily applied in our context, whereas our approach could be used for GUI test repair as well. Another difference between our approach and some of these techniques is that GUITESTMIGRATOR is fully automated and does not require any human effort.

\subsection{GUI Test Generation}

In the domain of mobile apps, there a large body of research that focuses on GUI test generation techniques (e.g., [11, 12, 16, 25, 37, $38,56])$. This work is somehow related to ours, in that generating new tests for an app is an alternative to migrating them from other apps. There is also more recent work that focuses on exploiting commonalities between apps to generate more effective GUI tests and that is more closely related to our approach (e.g., [14, 39, 40, 46]). Polariz [39] generates test scripts from crowd-generated tests by extracting cross-app reusable higher-level event sequences. Augusto [40] generates semantic UI tests based on popular features. Rau, Hotzkow, and Zeller [46] also propose the idea of generating efficient GUI tests by learning from tests of other apps. Also in this case, our work differs from these approaches because GUITESTMIGRATOR migrates test cases between apps to make the assessment of mobile app coding assignments more efficient, rather than performing GUI test generation.

\section{CONCLUSION AND FUTURE WORK}

A large number of students worldwide take online courses, resulting in scalability challenges when assessing student assignments. In this paper, we present GUITESTMigRATOR, a technique that tackles the specific problem of assessing mobile app development assignments. Given a set of apps that implement the same specification, but can have completely different user interfaces, GUITESTMIGRATOR allows for developing tests for one of these apps and automatically migrating these tests to the other apps, thus dramatically reducing the burden of assessing the apps.

We implemented a prototype of GUITESTMigrator that supports Android apps and used it on three different sets of apps developed by students of an online graduate-level software engineering course during three different semesters. We believe that the initial results presented in the paper are encouraging and motivate additional research along several directions. GUITESTMIGRATOR was able to successfully migrate, on average, $78 \%$ of the test events from source to target apps (and of the remaining 22\%, 10\% did not actually have a counterpart in the target apps). Overall, this resulted in GUITESTMIgRATOR fully migrating $68 \%$ of the test scenarios and partially migrating $21 \%$ of them.

In future work, we plan to explore two main directions. First, we will investigate ways to extend our technique so that it can migrate test oracles (i.e., assertions) in addition to GUI events. Second, we will leverage our findings to generalize GUITESTMigrATor so that it can migrate test cases not only mobile apps developed based on the same specification, but also apps that share only part of their functionality (e.g., apps in a given domain, which typically have common features).

As we describe in a recent poster [14], our long-term vision is to enable the development of a test store that operates in parallel to the app store: developers could submit their apps to the store, and the store would analyze them and look for similar apps, migrate tests from these apps, and return the successfully migrated tests.

\section{ACKNOWLEDGMENTS}

This work was partially supported by NSF under awards CCF1161821 and CCF-1563991. 


\section{REFERENCES}

[1] 2018. Android Studio. https://developer.android.com/studio/.

[2] 2018. Class Central Universities. https://www.class-central.com/universities.

[3] 2018. Espresso. https://google.github.io/android-testing-support-library/.

[4] 2018. Gradescope. https://www.gradescope.com.

[5] 2018. GUI Test Migration. https://sites.google.com/view/testmigration/.

[6] 2018. Mobile App Courses. https://learntocodewith.me/reviews mobile-app-courses/.

[7] 2018. UI Automator. https://developer.android.com/training/testing/ ui-automator/.

[8] 2018. Vocareum. https://www.vocareum.com.

[9] 2018. XML Path Language. https://www.w3.org/TR/xpath-30/.

[10] Kirsti Ala-Mutka. 2005. A Survey of Automated Assessment Approaches for Programming Assignments. Computer Science Education 15 (06 2005), 83-102.

[11] Domenico Amalfitano, Anna Rita Fasolino, Porfirio Tramontana, Salvatore De Carmine, and Atif M. Memon. 2012. Using GUI Ripping for Automated Testing of Android Applications. In Proceedings of the 27th IEEE/ACM International Conference on Automated Software Engineering (ASE 2012). ACM, New York, NY, USA, 258-261.

[12] Saswat Anand, Mayur Naik, Mary Jean Harrold, and Hongseok Yang. 2012. Automated Concolic Testing of Smartphone Apps. In Proceedings of the ACM SIGSOFT 20th International Symposium on the Foundations of Software Engineering (FSE '12). ACM, New York, NY, USA, Article 59, 11 pages.

[13] S. M. Arifi, I. N. Abdellah, A. Zahi, and R. Benabbou. 2015. Automatic program assessment using static and dynamic analysis. In 2015 Third World Conference on Complex Systems (WCCS). IEEE, Washington, DC, USA, 1-6.

[14] Farnaz Behrang and Alessandro Orso. 2018. Poster: Automated Test Migration for Mobile Apps. In Proceedings of the 40th International Conference on Software Engineering Companion (ICSE '18). ACM, New York, NY, USA, 384-385.

[15] Brenda Cheang, Andy Kurnia, Andrew Lim, and Wee-Chong Oon. 2003. On Automated Grading of Programming Assignments in an Academic Institution. Comput. Educ. 41, 2 (Sept. 2003), 121-131.

[16] Wontae Choi, George Necula, and Koushik Sen. 2013. Guided GUI Testing of Android Apps with Minimal Restart and Approximate Learning. SIGPLAN Not. 48, 10 (Oct. 2013), 623-640.

[17] Shauvik Roy Choudhary, Dan Zhao, Husayn Versee, and Alessandro Orso. 2011 WATER: Web Application TEst Repair. In Proceedings of the First International Workshop on End-to-End Test Script Engineering (ETSE '11). ACM, New York, NY, USA, 24-29.

[18] Brett Daniel, Qingzhou Luo, Mehdi Mirzaaghaei, Danny Dig, Darko Marinov, and Mauro Pezzè. 2011. Automated GUI Refactoring and Test Script Repair. In Proceedings of the First International Workshop on End-to-End Test Script Engineering (ETSE '11). ACM, New York, NY, USA, 38-41.

[19] Paul Denny, Andrew Luxton-Reilly, Ewan Tempero, and Jacob Hendrickx. 2011. CodeWrite: Supporting Student-driven Practice of Java. In Proceedings of the 42Nd ACM Technical Symposium on Computer Science Education (SIGCSE '11). ACM, New York, NY, USA, 471-476.

[20] Danny Dig and Ralph Johnson. 2006. Automated Upgrading of Component-based Applications. In Companion to the 21st ACM SIGPLAN Symposium on Objectoriented Programming Systems, Languages, and Applications (OOPSLA '06). ACM, New York, NY, USA, 675-676.

[21] Stephen H. Edwards and Manuel A. Perez-Quinones. 2008. Web-CAT: Automatically Grading Programming Assignments. In Proceedings of the 13th Annual Conference on Innovation and Technology in Computer Science Education (ITiCSE '08). ACM, New York, NY, USA, 328-328.

[22] C. Fu, M. Grechanik, and Q. Xie. 2009. Inferring Types of References to GUI Objects in Test Scripts. In 2009 International Conference on Software Testing Verification and Validation. IEEE, New York, NY, USA, 1-10.

[23] Z. Gao, Z. Chen, Y. Zou, and A. M. Memon. 2016. SITAR: GUI Test Script Repair. IEEE Transactions on Software Engineering 42, 2 (Feb 2016), 170-186.

[24] Mark Grechanik, Qing Xie, and Chen Fu. 2009. Maintaining and Evolving GUIdirected Test Scripts. In Proceedings of the 31st International Conference on Software Engineering (ICSE '09). IEEE Computer Society, Washington, DC, USA, 408-418.

[25] Shuai Hao, Bin Liu, Suman Nath, William G.J. Halfond, and Ramesh Govindan. 2014. PUMA: Programmable UI-automation for Large-scale Dynamic Analysis of Mobile Apps. In Proceedings of the 12th Annual International Conference on Mobile Systems, Applications, and Services (MobiSys '14). ACM, New York, NY, USA, 204-217.

[26] Jack Hollingsworth. 1960. Automatic Graders for Programming Classes. Commun. ACM 3, 10 (Oct. 1960), 528-529.

[27] Si Huang, Myra B. Cohen, and Atif M. Memon. 2010. Repairing GUI Test Suites Using a Genetic Algorithm. In Proceedings of the 2010 Third International Conference on Software Testing, Verification and Validation (ICST '10). IEEE Computer Society, Washington, DC, USA, 245-254.

[28] Petri Ihantola, Tuukka Ahoniemi, Ville Karavirta, and Otto Seppälä. 2010. Review of Recent Systems for Automatic Assessment of Programming Assignments. In Proceedings of the 10th Koli Calling International Conference on Computing Education Research (Koli Calling '10). ACM, New York, NY, USA, 86-93.
[29] Katy Jordan. 2014. Initial trends in enrolment and completion of massive open online courses. International Review of Research in Open and Distance Learning 15, 1 (February 2014), 133-160.

[30] G. Juniwal, A. DonzÃl', J. C. Jensen, and S. A. Seshia. 2014. CPSGrader: Synthesizing temporal logic testers for auto-grading an embedded systems laboratory. In 2014 International Conference on Embedded Software (EMSOFT). IEEE, New York, NY, USA, 1-10.

[31] Andrew S. Lan, Divyanshu Vats, Andrew E. Waters, and Richard G. Baraniuk. 2015. Mathematical Language Processing: Automatic Grading and Feedback for Open Response Mathematical Questions. In Proceedings of the Second (2015) ACM Conference on Learning @ Scale (L@S '15). ACM, New York, NY, USA, 167-176.

[32] Hao Li, Bo-Jhang Ho, Bharathan Balaji, Yue Xin, Paul Martin, and Mani B. Srivastava. 2017. EmbedInsight: Automated Grading of Embedded Systems Assignments. CoRR abs/1703.04514 (2017), 1-10.

[33] Peng Li and Eric Wohlstadter. 2008. View-based Maintenance of Graphical User Interfaces. In Proceedings of the 7th International Conference on Aspect-oriented Software Development (AOSD '08). ACM, New York, NY, USA, 156-167.

[34] Sihan Li, Xusheng Xiao, Blake Bassett, Tao Xie, and Nikolai Tillmann. 2016. Measuring Code Behavioral Similarity for Programming and Software Engineering Education. In Proceedings of the 38th International Conference on Software Engineering Companion (ICSE '16). ACM, New York, NY, USA, 501-510.

[35] X. Li, N. Chang, Y. Wang, H. Huang, Y. Pei, L. Wang, and X. Li. 2017. ATOM: Automatic Maintenance of GUI Test Scripts for Evolving Mobile Applications. In 2017 IEEE International Conference on Software Testing, Verification and Validation (ICST). IEEE, New York, NY, USA, 161-171.

[36] A. D. Lucia, R. Francese, G. Scanniello, G. Tortora, and N. Vitiello. 2006. A Strategy and an Eclipse Based Environment for the Migration of Legacy Systems to Multi-tier Web-based Architectures. In 2006 22nd IEEE International Conference on Software Maintenance. IEEE, New York, NY, USA, 438-447.

[37] Aravind Machiry, Rohan Tahiliani, and Mayur Naik. 2013. Dynodroid: An Input Generation System for Android Apps. In Proceedings of the 2013 9th foint Meeting on Foundations of Software Engineering (ESEC/FSE 2013). ACM, New York, NY, USA, 224-234.

[38] Riyadh Mahmood, Nariman Mirzaei, and Sam Malek. 2014. EvoDroid: Segmented Evolutionary Testing of Android Apps. In Proceedings of the 22Nd ACM SIGSOFT International Symposium on Foundations of Software Engineering (FSE 2014). ACM, New York, NY, USA, 599-609.

[39] K. Mao, M. Harman, and Y. Jia. 2017. Crowd intelligence enhances automated mobile testing. In 2017 32nd IEEE/ACM International Conference on Automated Software Engineering (ASE). ACM, New York, NY, USA, 16-26.

[40] Leonardo Mariani, Mauro PezzÃíl, and Daniele Zuddas. 2018. Augusto: Exploiting popular functionalities for the generation of semantic GUI tests with oracles. In Proceedings of the 40th International Conference on Software Engineering (ICSE '18). ACM, New York, NY, USA, 280-290.

[41] Atif M. Memon. 2008. Automatically Repairing Event Sequence-based GUI Test Suites for Regression Testing. ACM Trans. Softw. Eng. Methodol. 18, 2, Article 4 (Nov. 2008), 36 pages.

[42] Atif M. Memon and Mary Lou Soffa. 2003. Regression Testing of GUIs. SIGSOFT Softw. Eng. Notes 28, 5 (Sept. 2003), 118-127.

[43] George A. Miller. 1995. WordNet: A Lexical Database for English. Commun. ACM 38, 11 (Nov. 1995), 39-41.

[44] Adi Omari, Sharon Shoham, and Eran Yahav. 2016. Cross-supervised Synthesis of Web-crawlers. In Proceedings of the 38th International Conference on Software Engineering (ICSE '16). ACM, New York, NY, USA, 368-379.

[45] Adi Omari, Sharon Shoham, and Eran Yahav. 2017. Synthesis of Forgiving Data Extractors. In Proceedings of the Tenth ACM International Conference on Web Search and Data Mining (WSDM '17). ACM, New York, NY, USA, 385-394.

[46] Andreas Rau, Jenny Hotzkow, and Andreas Zeller. 2018. Poster: Efficient GUI test generation by learning from tests of other apps. In Proceedings of the 40th International Conference on Software Engineering Companion (ICSE '18). ACM, New York, NY, USA, 370-371.

[47] Dhawal Shah. 2017. Class Central Providers. https://www.class-central.com/ report/mooc-providers-list/.

[48] Heng Tao Shen, Beng Chin Ooi, and Kian-Lee Tan. 2000. Giving Meanings to WWW Images. In Proceedings of the Eighth ACM International Conference on Multimedia (MULTIMEDIA '00). ACM, New York, NY, USA, 39-47.

[49] Mark Sherman, Sarita Bassil, Derrell Lipman, Nat Tuck, and Fred Martin. 2013. Impact of Auto-grading on an Introductory Computing Course. f. Comput. Sci. Coll. 28, 6 (June 2013), 69-75.

[50] Rishabh Singh, Sumit Gulwani, and Armando Solar-Lezama. 2013. Automated Feedback Generation for Introductory Programming Assignments. In Proceedings of the 34th ACM SIGPLAN Conference on Programming Language Design and Implementation (PLDI '13). ACM, New York, NY, USA, 15-26.

[51] Thabet Slimani. 2013. Description and Evaluation of Semantic Similarity Measures Approaches. International Journal of Computer Applications Vol 80 (10 2013), 25-33.

[52] S. Staiger. 2007. Static Analysis of Programs with Graphical User Interface. In 11th European Conference on Software Maintenance and Reengineering (CSMR'07). 
IEEE, New York, NY, USA, 252-264.

[53] Hao-Chuan Wang, Chun-Yen Chang, and Tsai-Yen Li. 2008. Assessing creative problem-solving with automated text grading. Computers and Education 51, 4 (2008), 1450 - 1466

[54] Zhibiao Wu and Martha Palmer. 1994. Verbs Semantics and Lexical Selection. In Proceedings of the 32Nd Annual Meeting on Association for Computational Linguistics (ACL '94). Association for Computational Linguistics, Stroudsburg, PA, USA, 133-138.

[55] Shengqian Yang. 2015. Static Analyses of GUI Behavior in Android Applications. Ph.D. Dissertation. The Ohio State University.
[56] Wei Yang, Mukul R. Prasad, and Tao Xie. 2013. A Grey-box Approach for Automated GUI-model Generation of Mobile Applications. In Proceedings of the 16th International Conference on Fundamental Approaches to Software Engineering (FASE'13). Springer-Verlag, Berlin, Heidelberg, 250-265.

[57] Sai Zhang, Hao Lü, and Michael D. Ernst. 2013. Automatically Repairing Broken Workflows for Evolving GUI Applications. In Proceedings of the 2013 International Symposium on Software Testing and Analysis (ISSTA 2013). ACM, New York, NY, USA, 45-55. 